\title{
Session 10: Applied technologies - II
}

\author{
Friday 10 October 2003. Moderators: Larry Green and Mark Glassy
}

[10.40-11.10]

\author{
Immunotoxicotherapy with human anti-drug \\ antibodies: Digitalis and cocaine toxicity therapies \\ W.J. Ball ${ }^{\mathrm{a}, *}$, S. Paula ${ }^{\mathrm{a}}$, C. Farr ${ }^{\mathrm{a}}$, D. Williams ${ }^{\mathrm{b}}$, W.J. \\ $\mathrm{Welsh}^{\mathrm{c}}$ and A.B. Norman ${ }^{\mathrm{a}}$ \\ ${ }^{a}$ University of Cincinnati College Med., Cincinnati, \\ OH 45267, USA \\ ${ }^{\mathrm{b}}$ Medarex, Inc. Milpitas, CA, 95035, USA \\ ${ }^{\mathrm{c}}$ R.W. Johnson Med. School, Piscataway, NJ, 08845, \\ USA \\ *Tel.: +1 513558 2388; E-mail: William.Ball@uc. \\ $e d u$
}

Abstract: The potentials for the use of drug-specific antibodies or Fab fragments to detoxify patients suffering from overdoses occurring during therapeutic treatment or from the self-administration of drugs of abuse have long been recognized. However, the inherent difficulties resulting from the use of foreign-species proteins such as sheep polyclonal antibodies (Abs) or mouse monoclonal antibodies (mAbs) have restricted their use. Immunization of transgenic mice with "humanized" humoral systems [1,2] with hapten-carrier conjugates has allowed us to generate hybridoma cell lines that secrete human sequence mAbs of high affinity and specificity against the low molecular weight drugs digoxin and cocaine [3,4]. Determination of the fine-binding specificities of these mAbs has enabled us to develop 3-dimensional molecular models of their mechanisms of drug-mAb binding. These models have the potential to direct the modification of these mAbs to achieve improved affinities or broadened specificities as needed for a better clinical product.

Introduction: The use of current technologies that enable the generation of human sequence mAbs from transgenic or human PBL-engrafted mice, Ab or Fab phage display and $\mathrm{ScFv}$ libraries rather than foreign species-derived polyclonal Abs is greatly facilitating the development of new immunological treatments. But, the clinical use of anti-drug Abs also requires a thorough understanding of each $\mathrm{Ab}$ and the drug's pharmacokinetic (PK) characteristics. In these studies we report the development and characterization of the binding properties of human mAbs-directed against digoxin and cocaine. Since digoxin has a slow rate of elimination, a very narrow therapeutic index for its safe usage and undergoes little in vivo metabolism, its overdose treatment strategy requires high affinity Fab fragments that can bind free drug, reverse its toxicity and remove it through drug-Fab complex excretion in urine. In contrast, mAbs used for cocaine abuse relapse treatments require a high-specificity for cocaine versus its metabolites and a long circulation half-life within the blood so they are consistently present prior to any drug administration. They must serve as an alternative receptor for cocaine binding, thus altering the drug's PK and entry to the brain. Further, the rates of drug distribution $\left(\mathrm{k}_{\mathrm{o}}\right)$, elimination $\left(\mathrm{k}_{\mathrm{el}}\right)$, metabolism, volume of distribution $\left(\mathrm{V}_{\mathrm{d}}\right)$, and the extent of its nonspecific plasma protein binding all influence the requirements for successful immunotherapy. These issues as well as our determinations of the binding properties of our antidigoxin and anti-cocaine human mAbs are presented below.

Methods and materials: Generation of human sequence $m A b s$. The mice $[(\mathrm{C} 57 \mathrm{BL} / 6 \mathrm{~J}) \times(\mathrm{CBA} / \mathrm{J}) \mathrm{F} 2]$ used to generate the human antibody responses have undergone genetic modifications that produce doubletransgene/double-deletion mice designated by Medarex as the HuMAb-Mouse ${ }^{\circledR}$ have been previously described $[1,2]$. Drug-directed human $\operatorname{IgG}$ responses were elicited with i.p. injection of digoxin-KLH and benzoylecgonine-KLH conjugates suspended in CFA, followed by weekly or biweekly injections in IFA. Mouse splenic lymphocytes were fused with P3X63Ag8.653 nonsecreting mouse myeloma cells with the hybridomas grown and then selected for secretion of hapten-specific human sequence immunoglobulins by ELISA. Isotype analysis of the selected mAbs was achieved by ELISA using species and $\mathrm{H}$ or $\mathrm{L}$ chainspecific immunoreagents. NH2-terminal amino acid 
and genomic DNA sequencing of the mAb variable regions was also carried out.

Determination of relative binding affinities (RBAs) and affinity $\left(K_{d}\right.$ and $\left.K_{i}\right)$ constants.

The relative binding specificies of the mAbs for a large collection of drug derivatives was accomplished through the use of a competition binding ELISA that has been described [3,5]. Affinity constant values for $\mathrm{mAb}$ binding of compounds were obtained using a radioligand binding assay that employs a double-Ab precipitation technique and the collection of the $\left[{ }^{3} \mathrm{H}\right]$ ligand$\mathrm{mAb}-(\mathrm{Ab})_{\mathrm{x}}$ complexes on glass-fiber filters for quantification.

$3 D$ modeling of ligand structures and binding interactions with $m A b$. The $3 \mathrm{D}$ structures of the collections of digoxin or cocaine-based ligands were first modeled from x-ray crystal structures of digoxin or cocaine and then the modified structures were drawn and energy minimized using Sybyl software from Tripos, Inc. The 3D quantitative structure-activity (3D-QSAR) relationship models that identify those regions of the ligands contributing to the drug-mAb binding interactions were generated using the QSAR module of Sybyl.

Results and discussion: Antibody generation. Seven hybridoma cell lines secreting anti-digoxin mAbs were successfully cloned from the spleenocytes of two of six animals immunized with a digoxin-KLH conjugate and undergoing cell fusion. The generation of anticocaine mAbs was more problematic with benzoylecgonine (BE) being coupled to four different proteins that were tried as immunogens. Following immunization with these conjugations, a number of unsuccessful cell fusions were performed. However, a cell fusion using spleenocytes from an animal immunized with a BE-KLH conjugate produced five hybridoma cell lines that secreted anti-cocaine mAbs. Amongst these, the hybridoma cell designated, 2E2 was selected for further cloning and characterization of its anti-cocaine mAb.

Characterization of $m A b$ binding avidities, affinities and specificities. Hybridoma cell lines were conditioned to grow as T-flask cultures and mAb present in spent medium was used for initial $\mathrm{mAb}$ specificity testing. mAb concentrations were determined by capturing mAbs from solution with plate adsorbed humanspecific IgGs. Ab dilution-binding curves then gave avidity values of about $1-5 \mathrm{nM}$ for all $\mathrm{mAbs}$. The mAbs were also found to bind to their appropriate hapten as conjugated to several different proteins while showing no binding to carrier proteins.

mAbs produced from mouse ascites fluid were purified using proteinA-column chromato-graphy for de-

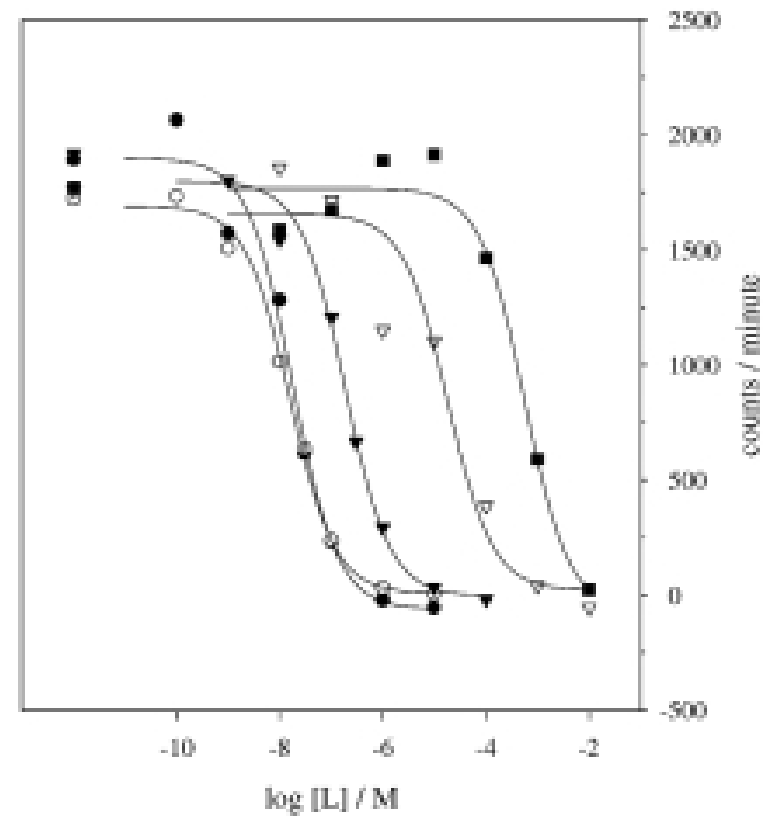

Fig. 1. Inhibition curves, cocaine $(\bullet)$ and derivatives (mAb 2E2).

Table 1

Cardiac glycoside affinities

\begin{tabular}{llll}
\hline Drugs & $7 \mathrm{~F} 2$ & $1 \mathrm{~B} 3$ & $11 \mathrm{E} 6$ \\
\hline Digoxin* & $2.5 \mathrm{nM}$ & $3.1 \mathrm{nM}$ & $3.1 \mathrm{nM}$ \\
Digitoxin* & 7 & 0.6 & 8 \\
Digitoxigenin & 78 & 4,039 & 34 \\
Neriifolin & 215 & 126 & 33 \\
Bufalin & 19,623 & 717 & 2,990 \\
Oleandrin & 10,879 & 252 & 73,461 \\
Digitoxigenin(mono) & 205 & 22 & 23 \\
Thevetin B & 189 & 280 & 16 \\
Evomonoside & 172 & 32 & 23 \\
\hline
\end{tabular}

tailed characterization. Radioligand binding experiments indicated that anti-digoxin mAbs designated, 1B3, 7F2, 11E6 and 5C2 had $\mathrm{K}_{\mathrm{d}}$ values for digoxin of $3.1,2.5,4.5$ and $22 \mathrm{nM}$, respectively. The anti-cocaine $\mathrm{mAb} 2 \mathrm{E} 2$ bound cocaine with a $\mathrm{K}_{\mathrm{d}}$ value of $4 \mathrm{nM}$. Interestingly, while in most cases compounds closely related to the original immunogen bound well although, as expected, with somewhat poorer nM affinities, antidigoxin mAb 1B3 was found to bind digitoxin $\left(\mathrm{K}_{\mathrm{d}}\right.$, $0.6 \mathrm{nM})$ better than digoxin, and anti-cocaine mAb $2 \mathrm{E} 2$ binds both cocaethylene ( $3 \mathrm{nM})$ and cocaine propylester (1 nM) better than cocaine (Fig. 1).

Development of 3-dimensional quantitative structureactivity relationship (3D-QSAR) models for $m A b$ binding. The fine-binding specificities or relative binding affinities (RBAs) of the mAbs for a large collection (56 cardiotonic and hormonal steroids; 38 cocaine analogues) of ligands were determined with a competition 

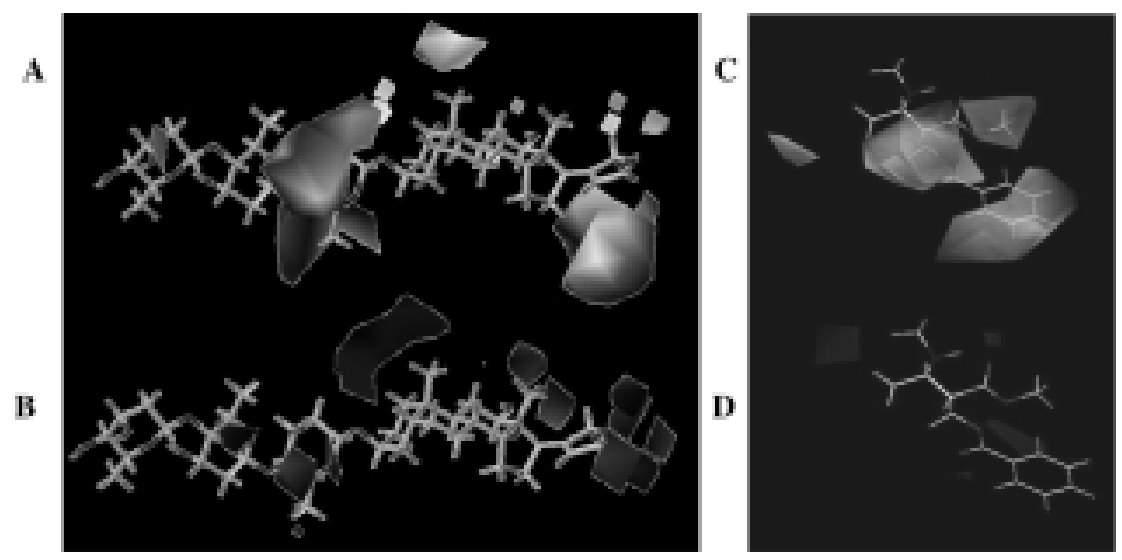

Fig. 2. 3D structure-activity contour models of drug:mAb interactions. A) Steric, B) Electrostatic interactions of digoxin with mAb 1B3; C) Steric, D) Electrostatic interactions of cocaine with mAb 2E2.

ELISA that used digoxin or cocaine as the $\mathrm{IC}_{50}$ standard for inhibition of $\mathrm{mAb}$ binding to a hapten-protein conjugate $[4,6]$. Simple inspection of the $\mathrm{IC}_{50}$ ratios for the anti-digoxin mAbs (Table 1, mAbs, 1B3, 7F2, 11E6) demonstrated that: cardiac glycoside sugars generally play little role in binding, only mAb $1 \mathrm{~B} 3$ required an asugar attached to the steroid moiety; the conformation of the steroid rings was not critical but the addition of multiple hydroxyl groups to the ring (ouabain) dramatically lowers binding; the five-membered, unsaturated lactone ring is also critical for $\mathrm{mAb}$ recognition. For anti-cocaine mAb 2E2 the ELISAs indicated that it is highly sensitive to stereochemistry of cocaine, further the removal of the $\mathrm{C} 2$ methyl-ester's methyl group reduces binding 10-fold while the phenyl group plays a pivotal role. Its removal decreases binding more than 2,000-fold.

Comparative molecular field analysis (CoMFA) was then used to correlate steric (van der Waals) and electrostatic (Coulombic) energies between the test compounds and a carbon atom probe representing the $\mathrm{mAb}$ environment about the ligands and their affinities in order to develop the 3D-contour plots such as shown in Figs 2(A)-(D), that identify the regions about the ligands where a change in the steric or electrostatic field parameters (ligand structure) affects ligand affinity. These analyses indicated that for digitalis binding to the mAbs [4], electrostatic interactions (60\%) predominate while for cocaine binding $[4,6]$ steric interactions (77\%) dominate. In the 3D-models (Fig. 2, mAbs; $1 \mathrm{~B} 3 \& 2 \mathrm{E} 2$ ), the green and yellow regions (Figs 3(A) and $(\mathrm{C})$ ) indicate areas where increased steric bulk of either ligand or receptor increases or decreases, respectively, binding affinity. In Figs 3(B) and (D) the red and blue areas indicate regions about ligand where increased negative or positive partial charges, respectively, in the ligand increases binding affinity. These 3D-QSAR maps not only indicate the structural regions or locations of important drug-mAb interactions but can map out the size and shape of mAb binding sites and when coupled with computer-aided protein structure modeling of the mAbs' Fv regions facilitate the identification of amino acid residues critical for the binding affinity and specificity. Modification of these residues has the protential for producing mAbs with improved affinities and specificities or broadened specificities as needed for a better clinical product.

Finally, we should point that the efficacy of detoxification for any $\mathrm{Ab}$ will not depend solely upon the mAb's binding affinity and specificity but also upon the in vivo pharmacokinetics of the drug being targeted as they affect drug-mAb interactions.

\section{References}

[1] N. Lonberg, L. Taylor, F. Harding, M. Trounstine, K. Higgins and G. Sequar et al., Nature 368 (1994), 856-859.

[2] D. Fishwild, S. O'Donnell, T. Bengoechea, D. Hudson and K. Harding, Nature Biotechnology 14 (1996), 845-851.

[3] W. Ball, R. Kasturi, P. Dey, M. Tabet, S. O'Donnell, D. Hudson and D. Fishwild, J. Immunol. 163 (1999), 2291-2298.

[4] S. Paula, M. Tabet, C. Farr, S. Keenan, W. Welsh, A. Norman and W. Ball, Biophys. J. 84(2) (2003), 2456.

[5] C. Farr, M. Tabet, D. Fishwild, X. Wang, A. Nair, W. Welsh and W. Ball, J. Med. Chem. 45 (2002), 3257-3270.

[6] S. Paula, M. Tabet, S. Keenan, W. Welsh and W. Ball, J. Mol. Biol. 325 (2003), 515-530. 


\section{[11.10-11.30]}

\section{PER.C6 $^{\mathrm{TM}}$ as production platform for human monoclonal antibodies \\ Bram Bout \\ Crucell, Leiden, The Netherlands}

PER.C6 ${ }^{\mathrm{TM}}$, an immortalized human cell line, was developed as a production platform for human monoclonal antibodies. In straight batch cultures, more than $1 \times 10^{7}$ viable cells per $\mathrm{ml}$ and $0.6-1.0 \mathrm{~g}$ per $\mathrm{L}$ are obtained. Daily medium exchange results in $3 \times 10^{7}$ viable cells per $\mathrm{ml}$ and $>0.5 \mathrm{~g} / \mathrm{L} / 24 \mathrm{~h}$. The glycans of monoclonal antibodies produced by PER.C6 ${ }^{\mathrm{TM}}$ are comparable to glycans found on human serum IgG. The high productivity, scalability and glycosylation make PER.C6 ${ }^{\mathrm{TM}}$ a cost-effective platform for the production of monoclonal antibodies with human characteristics.

\section{[11.30-12.00]}

\section{Overcoming immunogenicity by identification and removal of $\mathbf{T}$ cell epitopes \\ Frank Carr}

Biovation Ltd, Aberdeen Scince Park, Scotland, UK

Immunogenicity associated with therapeutic antibodies and proteins has been highlighted recently as a cause of serious complications and deaths. Even therapeutic antibodies engineered to contain human sequence, such as the chimaeric antibody, Infliximab, or the humanised antibody CAMPATH-1H, have been shown to be immunogenic.

Therapeutic antibodies may be derived from any of several sources including phage display, human Ig transgenic mice, xenotransplanted mice, or others, but in each case it is possible that these can elicit an immune response in certain patients. This is due to the process of affinity maturation during antibody generation that inevitably results in antibodies to which patients may not be naturally tolerant. A sustained and limiting antiglobulin response to therapeutic antibodies is a CD4 ${ }^{+}$ $\mathrm{T}$-cell dependent process typical of any foreign protein. Underlying this process is the presentation of Th peptide epitopes derived from the intracellular processing of the therapeutic antibody. We have reasoned that if the Th peptide were unable to interact with sufficient avidity or were conformationally barred from binding within the MHC class II peptide binding cleft, then the Th signals could not be established and the therapeutic antibody rendered non-immunogenic. This strategy is dependent on identification of the Th epitope and mutational modification of the protein sequence to compromise the MHC class II binding interaction.

We have focused on this approach in our laboratory to produce a number of antibody products in which $\mathrm{T}$ cell epitopes have been removed without detriment to their structure or function. Epitope identification has been made possible by the combined use of ex vivo human T-cell proliferation assays and a software tool termed Peptide Threading (PT). The PT analysis permits precise modelling of the effects of amino acid substitutions on the ability of a Th epitope sequence to interact with multiple different MHC class II allotypes present in the human population. This provides guidance in the design of engineered antibodies with a more favourable immunogenicity profile. One such antibody targets human TNF $\alpha$ and despite multiple V-region substitutions we have data demonstrating retention of all functional capabilities of the molecule with respect to TNF $\alpha$ neutralisation. Assays include competition experiments with recombinant TNF-receptor and cell surface bound $\mathrm{TNF} \alpha$; protection of $\mathrm{TNF} \alpha$-induced killing of WEHI cells and suppression of TNF $\alpha$-induced expression of ICAM-1 and IL-6 in HUVEC and human fibroblast cells respectively. Surface plasmon resonance data and other pre-clinical efficacy assays are also favourable. Critically, this modified antibody has been demonstrated to no longer stimulate the proliferation of human $\mathrm{T}$ cells in ex vivo human $\mathrm{T}$ cell proliferation assays. This antibody is therefore an attractive candidate for clinical development and these findings highlight the promise of this approach as a generalised scheme for improving the immunogenicity profiles of therapeutic antibodies and other proteins.

\section{[12.00-12.20]}

\author{
GENOVAC technology: A breakthrough in \\ generating functional antibodies against GPCRs \\ John Thompson and Stefan Lang \\ GENOVAC AG, Waltershofener Str. 17, D-79111 \\ Freiburg, Germany \\ URL: www.genovac.com
}

GENOVAC's technology has the best potential of all immunisation methods to produce antibodies that recognise native proteins and accordingly can be used in immunological methods investigating living cells. Its qualities render antibodies originating from genetic immunisation especially suitable as diagnostic and therapeutic agents. Together with the high specificity and affinity of the antibodies this is a prerequisite for the identification of the corresponding antigens in complex 
mixtures and environments such as blood or body tissues, where the targets are often present in low concentrations. The combination of all these properties renders it possible to use these antibodies in vivo as carriers of toxins, drugs, drug-activating enzymes, radioactive isotopes and photo-labels to target specific disease sites in the body.

Membrane-bound and secreted proteins are the most interesting targets for diagnosis and therapy. Secreted proteins often serve as target structures for diagnostics to avoid invasive techniques in acquiring test samples from patients. Therapeutics, on the other hand, are often directed against membrane-bound proteins which are extracellularly accessible from blood or lymph. Both classes of proteins create problems in the conventional protein and peptide immunisation since they are subject to pronounced post-translational modifications and characterised by strong hydrophobic properties, respectively.

GPCRs constitute the largest family of cell-surface molecules involved in signal transduction. These seven-transmembrane-domain receptors play key physiological roles and their dysfunction results in several diseases. GPCRs are the target of $50-70 \%$ of the current therapeutic agents on the market with annual worldwide sales exceeding US $\$ 30$ billion in 2001. The human genome project has identified approximately 150 GPCRs as potential new drug targets. Accordingly, members of the GPCR family represent up to $30 \%$ of the R\&D portfolio of many pharmaceutical companies. They are the main targets to identify novel approaches for pharmacological intervention with inhibitory or stimulatory agents. Data on the successful generation of monoclonal antibodies recognising native GPCRs and blocking their activity will be presented.

\section{[12.20-12.40]}

\section{A simple, low cost, high volume and concentration method for production of hybridoma antibody V. Mehrotra ${ }^{\mathrm{a}}$, V.L. Lahiri ${ }^{\mathrm{b}}$ and D.K. Hazra ${ }^{\mathrm{b}}$ ${ }^{a}$ Biochemistry Department, Himalayan Institute of Medical Sciences, Dehradun, India \\ ${ }^{a} S N$ Medical Sciences, Agra, India}

The hybridoma technology developed 30 years back known as invitro technology was very expensive with skilled manpower needed to operate them. Due to the high maintance, problems in animal house and obtaining for the hybridoma cells generated by an invitro procedure and selected for the use after screening of invitro-produced tissue culture supernatants. These su- pernatants should be of very high purity, free of contaminants and of high concentration for directly labeled with a marker. To fulfill these conditions we develop an ordinary glass-rolling chamber with dialysis tubes dipped in DMEM. The hybridoma culture cells between $6 \times 10^{5}$ cells $/ \mathrm{ml}$ with $5 \%$ FCS was added to tubes. The medium was gassed with $5 \% \mathrm{CO}_{2}$ daily and the apparatus was rolled.

It was observed that after 10 days the cell numbers increased without excessive degradation of the dead cells and the concentration was very high when compared with the ordinary cell culture method. The rolling motion caused the air bubble inside the tube to oscillate, which was to be an advantageous and impurities were effectively lower. The chambers were reusable and hold more than two different hybridoma cell lines. Therefore, this culture method is a useful alternative production of antibody with simple, low cost and high volume and concentration. This production can be easily adaptable to tissues culture laboratory routine and without the need of animals.

\section{[12.40-13.00]}

\section{Amylolytic activity and catalytic properties of IgM and IgG antibodies from patients with systemic lupus erithromatosis \\ Kirill N. Neustroev ${ }^{\mathrm{a}}$, Dina R. Ivanen ${ }^{\mathrm{a}}$, Anna A. Kulminskaya $^{\mathrm{a}}$, Harry Brumer III ${ }^{\mathrm{b}}$, Andrew N. Saveliev $^{\mathrm{c}}$ and Georgy A. Nevinsky ${ }^{\mathrm{d}}$ \\ ${ }^{a}$ Molecular and Radiation Biophysics Division, Petersburg Nuclear Physics Institute of Russsian Academy of Sciences, Gatchina, Russia \\ ${ }^{\mathrm{b}}$ Biophysics Department, St. Petersburg Technical University, St. Petersburg, Russia \\ ${ }^{\mathrm{c}}$ Department of Biotechnology, Royal Insitute of Technology (KTH), Stockholm Center for Physics, Astronomy, \& Biotechnology, Stockholm, Sweden ${ }^{\mathrm{d}}$ Novosibirsk Institute of Bioorganic Chemistry, Siberian Division of Russian Academy of Sciences, Novosibirsk, Russia}

Introduction: Natural human autoantibodies capable of catalyzing a variety of hydrolytic reactions were found and characterized in the last decade. Catalytically active antibodies (abzymes) that can hydrolyze peptides (vasoactive intestinal peptide) and proteins were detected in the sera of patients with bronchial asthma and rheumatoid arthritis [1,2]. The presence of DNA and RNA hydrolyzing IgGs was proved for such autoimmunity diseases like systemic lupus erithromatosis (SLE) [3,4] and multiple sclerosis [5]. 
One more type of hydrolytic activity that had been detected in fractions of human antibodies was amylolytic, which was expressed in ability of hydrolyzing $\alpha 1 \rightarrow 4$-glucosydic linkages in maltooligosaccharides and polymeric substrates, starch and glycogen. Abzymes with amylolytic activity hydrolyze $\alpha 1 \rightarrow 4$ glucosydic bonds in different oligomeric and polymeric substrates yielding generally maltose and maltotriose as major products of the reaction. Previously, we showed the presence of amylolytic activity in IgG and sIgA fractions from human milk of healthy women [6] and in immunoglobulins $\mathrm{G}$ isolated from the sera of patients suffering from cancer [7]. Further investigations revealed significantly higher level of the considered activity in IgM and IgG samples of MS patients [8]. Exo-amylolytic and a-glucosidase activities were also detected in $\operatorname{IgM}$ and $\operatorname{IgG}$ fractions isolated from the sera of patients with clinically confirmed diagnosis of SLE. Usage of oligomeric substrates modified with fluorogenic and chomatophoric groups enabled to study kinetic properties of SLE abzymes and detect differences in modes of action of $\operatorname{IgM}$ and $\mathrm{IgG}$ samples from different donors.

Purification of IgM and Ig G fractions with amylolytic activity: Since autoantibodies can form complexes with other proteins, and Ab-mediated catalysis is sometimes characterized by relatively low reaction rates, it is essential to prove that the catalytic activities of Ig fractions was not due to contaminating enzymes. Therefore, it was important to apply such a method of IgG and IgM purification, which allows separation of any minor admixture of human a-amylases and a-glucosidases. Subsequent chromatographies on a Mono Q, Superose 12, and Protein-A Sepharose or Protein-G Sepharose were optimal first steps of catalytic Ab purification. In a final step we used immunoaffinity chromatography of purified $\operatorname{IgG}$ and $\operatorname{IgM}$ preparations on adsorbents bearing antibodies to human $\operatorname{IgG}$ and $\operatorname{IgM}$, respectively as an additional effective tool for abzyme purification. Resulted IgM and IgG samples were homogenous according to SDS PAGE data, obtained using visualization with silver or Coumassi Brilliant Blue staining.

Application of strict criteria worked out previously in several studies concerning human hydrolytic abzymes [1-8] led us to conclusion that the observed amylolytic activity is an intrinsic property of human IgMs and IgGs and is not due to co-purifying human amylolytic enzymes. Firstly, the amylolytic activity coincided with the IgG or IgM protein peaks after Superose 12 chromatography in $6 \mathrm{M}$ guanidine chloride followed by renaturation by dialysis against neutral buffer
(Fig. 3(A)). Secondly, the amylolytic activity of the IgM and IgG fractions was totally suppressed by addition of immobilized antibodies to human IgM and IgG, respectively, and was recovered by elution with a low $\mathrm{pH}$ buffer. Thirdly, the amylolytic activity was not suppressed by incubation in $1 \mathrm{M}$ acetic acid for $2 \mathrm{~h}, 20^{\circ} \mathrm{C}$ (so-called "acid shock") and coincided with the IgM and $\mathrm{IgG}$ fractions after their subsequent gel-filtration on a Superose 12 column. Fourthly, after SDS-PAGE of a preparation of native IgG and IgM preparations and following renaturation, an in-gel assay showed amylolytic activity only in single protein bands corresponding to the complete IgG and IgM, respectively. Finally, Fab fragments obtained by papain digestion of corresponding IgGs showed practically the same level of the amylolytic activity than intreated IgGs. As shown on Fig. 3(B), a profile of the a-amylolytic activity obtained after gel-filtration of Fab fragments absolutely coincided with chromatography peak of the eluted Fab fraction.

Substrate specificity and hydrolytic properties: Fractions of IgMs from 42 patients with SLE were analyzed in this study. In all investigated cases, the specific amylolytic activity of isolated IgMs in the hydrolysis of pnitrophenyl maltohexaoside $\left(\mathrm{PNPG}_{6}\right)$ was in the range from 4 to $30 \times 10^{-2} \mathrm{U} / \mathrm{mg}$ (data for 20 of these patients were shown in Fig. 4), whereas polyclonal IgMs from healthy donors demonstrated specific a-amylolytic activity less than $0.1 \times 10^{-4} \mathrm{U} / \mathrm{mg}$. The levels of the a-amylolytic activity of IgG samples isolated from the sera of the same SLE patients were significantly lower except six samples (Fig. 4). It should be noted that average specific amylolytic activity of IgMs from SLE patients was about 2.5 times higher than that from MS patients. In comparison, average specific activity towards PNPG6 of IgG and sIgA samples from milk of healthy women was about $0.5 \times 10^{-3} \mathrm{U} / \mathrm{mg}$ and of IgGs from the sera of pregnant women was $0.1 \times 10^{-3} \mathrm{U} / \mathrm{mg}$. Therefore, fractions of IgMs isolated from blood of patients with definite SLE diagnosis appeared to be the most active among all other tested abzymes. Both IgM and $\mathrm{IgG}$ fractions from SLE patients are capable of hydrolyzing glycogen, starch and maltooligosaccharides of various lengths.

Analysis of kinetic properties of some IgGs and IgMs was performed on a reverse-phase HPLC column using $\mathrm{p}$-nitrophenyl maltopentaose $\left(\mathrm{PNPG}_{5}\right)$ as a substrate in assays of the a-amylolytic activity. The values of Michaelis-Menten constant $\left(K_{M}\right)$ and $k_{\text {cat }}$ determined from Lineweaver-Burk plots for several most active Abs are presented in Table 2. It should be men- 

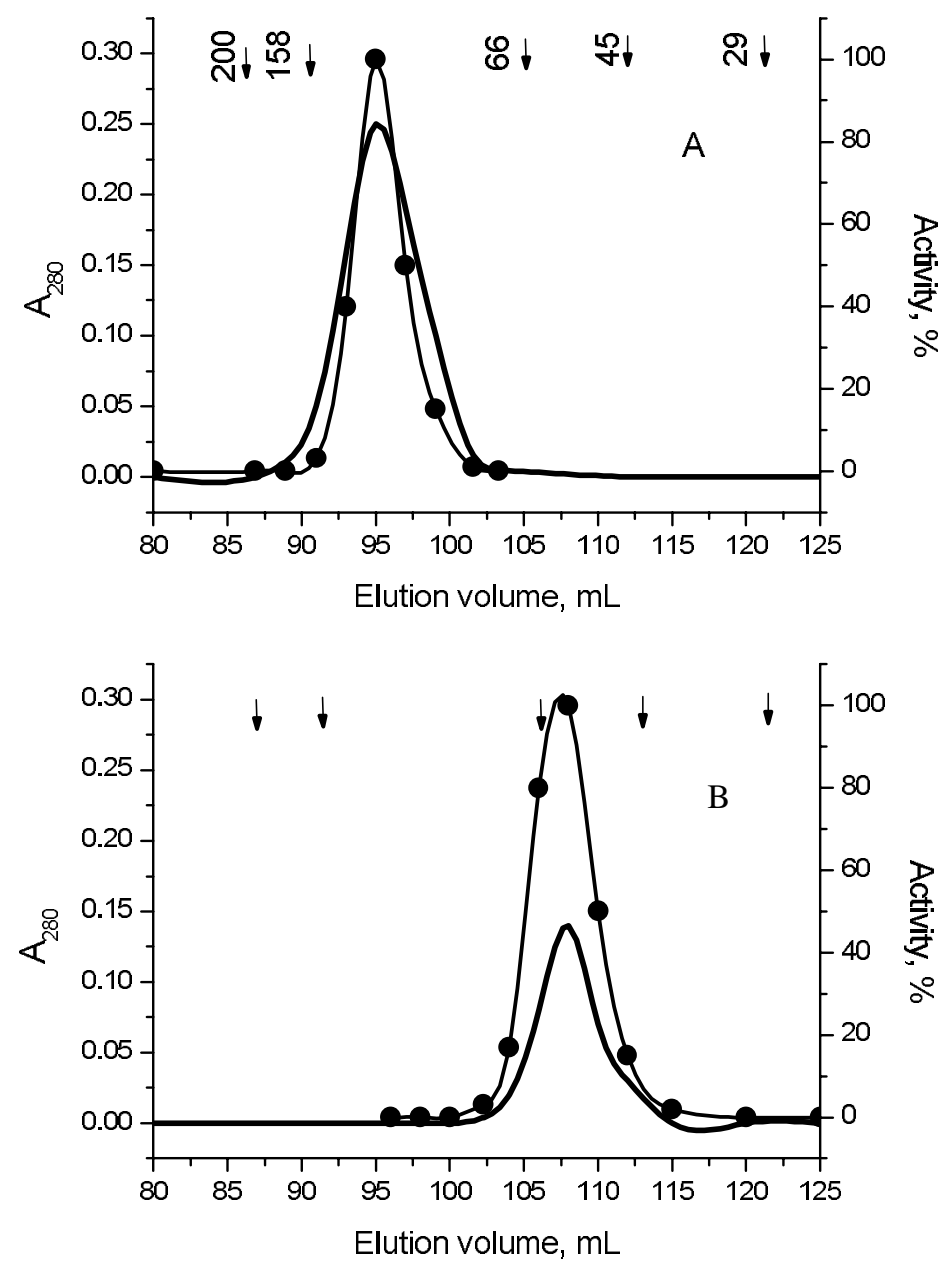

Fig. 3. Analytical gel-filtration of the amylolytic activity of an IgM fraction isolated from SLE patient number 2 (A) and the amylolytic activity of Fab fragment of the same IgM (B) performed on a Superose 12 column in a $6 \mathrm{M}$ guanidine chloride: •, activity; -, absorbance at $280 \mathrm{~nm}$.

tioned that the transition from untreated $\mathrm{IgG}_{3}$ and $\mathrm{IgG}_{7}$ ( $K_{M}=0.02$ and $0.12 \mathrm{mM}$, respectively) to its $\mathrm{Fab}$ fragments $\left(K_{M}=0.03\right.$ and $0.14 \mathrm{mM}$, respectively) does not practically change the $K_{M}$ and $k_{\text {cat }}$ values for the hydrolysis of $\mathrm{PNPG}_{5}$. In contrast to human amylases, the amylolytic activity of IgG and IgM fractions of catalytic Abs from all donors were not depended on $\mathrm{Cl}^{-}$ions. Values for $\mathrm{pH}$ optima of the $\mathrm{PNPG}_{5}$ hydrolysis for various SLE amylolytic abzymes were in the range of 6.0-8.0.

Mode of action of IgM and IgG fractions: The mode of action of $\operatorname{IgM}$ and $\operatorname{IgG}$ antibodies from 12 SLE patients was studied using 4-methylumbelliferyl maltooligosaccharides with d.p. 1-5 and PNP maltooligosaccharides with d.p. up to 12 as substrates with the following reverse-phase HPLC analysis of the products formed during the reaction. The ability of Abs to hydrolyze the above substrates was also examined by spectrophotometric techniques. The most clear difference is the presence of two main types of action modes for considered Abs: exo-amylolytic pattern without release of a free glucose and exo-amylolytic activity combined with exo-glycosidase one. The presence of a-glucosidase activity of some $\operatorname{IgG}$ and $\operatorname{IgM}$ samples was showed by the liberation of a free glucose from MeUmb- and PNP-glucosides. The specific activities of some IgM fractions from SLE patients in hydrolysis of maltohexaose and glycogen differ widely that indicates a variety of modes of action of these Abs and serves as an additional evidence for the presence of a-amylolytic activity in SLE antibodies. Analysis of IgG and IgM fractions from the sera of different SLE patients shows quite major differences in the mode of action in hydrolysis of different substrates. Only few antibody samples possess a-glucosidase activity giving a glucose as a product. One could only explain such 


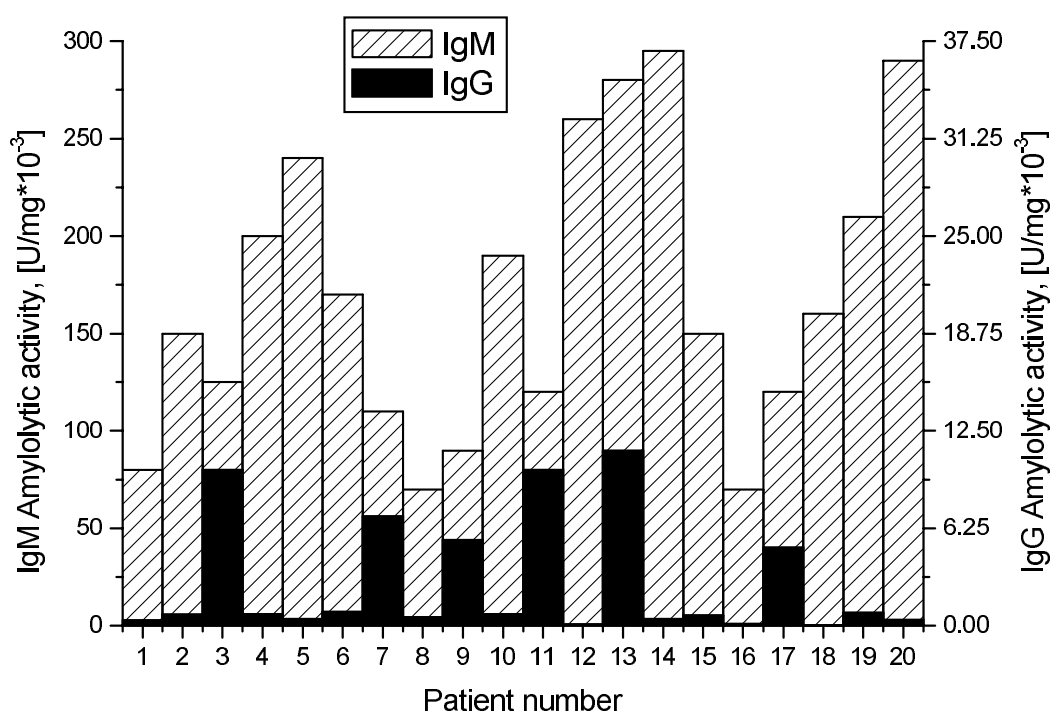

Fig. 4. A selectiv set of values for the specific a-amylolytic activities of IgMs and IgGs isolated from the same SLE patients in the hydrolysis of PNP maltohexaose.

Table 2

Kinetic parameters of hydrolysis of PNP maltopentaose catalyzed by several different preparations of IgM

\begin{tabular}{cccr}
\hline Fraction of immunoglobulin* & $K_{M}, \mathrm{mM}$ & $k_{\text {cat }}$ & \multicolumn{1}{c}{$k_{\text {cat }} / K_{M}$} \\
\hline $\mathrm{IgM}_{3}$ & $0.02 \pm 0.001$ & $290 \pm 3$ & $14500 \pm 3$ \\
$\mathrm{IgG}_{3}$ & $0.22 \pm 0.01$ & $205 \pm 3$ & $930 \pm 3$ \\
$\mathrm{IgM}_{5}$ & $0.44 \pm 0.01$ & $805 \pm 3$ & $1830 \pm 3$ \\
$\mathrm{IgM}_{6}$ & $0.36 \pm 0.01$ & $610 \pm 3$ & $1700 \pm 3$ \\
$\mathrm{IgM}_{12}$ & $0.12 \pm 0.01$ & $890 \pm 3$ & $7420 \pm 3$ \\
\hline
\end{tabular}

${ }^{*}$ Superscripts correspond to patient numbers.

differences in samples, which were isolated by a standard procedure, by the presence of contaminating enzymes with different types of amylolytic activity, a hypothesis which we believe is very unlikely. The reason of arising of hydrolytic activity in human autoantibodies in cases of autoimmunity pathologies is unclear for now. However, a-amylolytic assays of IgM fractions using fluorogenic substrates might serve as an method for detection SLE.

\section{References}

[1] S. Paul, D.J. Volle, C.M. Beach, D.R. Johnson, M.J. Powell and R.J. Massey, Catalytic hydrolysis of vasoactive intestinal peptide by human autoantibody, Science 244 (1989), 11581162.

[2] L. Li, S. Paul, S. Tyutyulkova, M.D. Kazatchkine and D. Kaveri, Catalytic activity of anti-thyroglobulin antibodies, J. Immunol. 154 (1995), 3328-3332.

[3] A.M. Shuster, G.V. Gololobov, O.A. Kvashuk, A.E. Bogomolova, I.V. Smirnov and A.G. Gabibov, DNA hydrolyzing autoantibodies, Science 256 (1992), 665-667.
[4] G.V. Gololobov, E.A. Chernova, D.V. Schourov, I.V. Smirnov, I.A. Kudelina and A.G. Gabibov, Cleavage of supercoiled plasmid DNA by autoantibody Fab fragment: application of the flow linear dichroism technique, Proc. Natl. Acad. Sci. USA 92 (1995), 254-257.

[5] G.A. Nevinsky and V.N. Buneva, Human catalytic RNAand DNA-hydrolyzing antibodies, J. Immunol. Methods 269 (2002), 235-249.

[6] A.N. Savel'ev, T.G. Kanyshkova, A.A. Kulminskaya, E.V. Buneva, E.V. Eneyskaya, M.V. Filatov, G.A. Nevinsky and K.N. Neustroev, Amylolytic activity of IgG and SIgA immunoglobulins from human milk, Clin. Chim. Acta 314 (2001), 141-152.

[7] A.N. Savel'ev, E.V. Eneyskaya, K.A. Shabalin, M.V. Filatov and K.N. Neustroev, Autoantibodies with amylolytic activity, Prot. Pept. Lett. 6 (1999), 179-184.

[8] A.N. Saveliev, D.R. Ivanen, A.A. Kulminskaya, N.A. Ershova, T.G. Kanyshkova, V.N. Buneva, A.S. Mogelnitskii, B.M. Doronin, O.O. Favorova, G.A. Nevinsky and K.N. Neustroev, Amylolytic activity of IgM and IgG antibodies from patients with multiple sclerosis, Immunol. Lett. 86(3) (2003), 291-297. 
[13.00-13.20]

Next-generation systems for screening and characterization of antibody-antigen interactions Anthony Chiulli*

Applied Biosystems, 35 Wiggins Avenue, Bedford, MA 01730, USA

* Contact Info: Anthony Chiulli, Product Manager, New Technologies, Cell Biology \& Functional Proteomics, Applied Biosystems, 35 Wiggins Avenue, Bedford, MA 01730, USA.

Tel.: +1 781280 5652; Fax: +1 781280 1414;

E-mail: achiulli@appliedbiosystems.com

Two systems have been made available for improving the development process of hybridoma and phage display-derived antibodies. Utilizing FMAT ${ }^{\circledR}$ system technology, the Applied Biosystems 8200 Cellular Detection System performs homogeneous assays using fluorescent based confocal imaging to scan the bottom of each well in a clear-bottom microplate. By scanning only a $100 \mu \mathrm{m}$ depth at the bottom of the wells, cells or beads can be clearly detected in relation to unbound fluorophores, eliminating the need for wash steps. This system combines the lower cost and higher throughput of ELISAs, with the functionality of flow cytometry, to improve the antibody screening process.

The Applied Biosystems 8500 Affinity Chip Analyzer is a new system that uses SpotMatrix Surface Plasmon Resonance technology to provide simultaneous measurement of real-time binding kinetics between an unlabeled analyte with up to 400 biomolecular targets spotted onto an affinity chip. Several antibody-based applications have been developed, including the characterization of monoclonal antibodies, peptide epitope mapping, and the identification of matched antibody pairs. These three applications provide researchers with valuable tools for biomolecular interaction analysis and antibody characterization.

Together, the 8200 and 8500 systems provide a more complete workflow solution for antibody screening and characterization. 